\title{
Correlation between protein YKL-40 and ultrasonographic findings in active knee osteoarthritis
}

\author{
Rositsa Karalilova1, Maria Kazakova², Anastas Batalov1, Victoria Sarafian²,3
}

${ }^{1}$ Department of Propaedeutic of Internal Diseases, Medical University, Clinic of Rheumatology, University Hospital "Kaspela", ${ }^{2}$ Department of Medical Biology, Faculty of Medicine, Medical University of Plovdiv, ${ }^{3}$ Technological Center for Emergency, Plovdiv, Bulgaria

\begin{abstract}
The aim of our study was to analyze the level of the glycoprotein YKL-40 in patients with active knee osteoarthritis (OA) and to search possible correlations with local inflammation and ultrasound (US) findings. Material and methods: A prospective study with fifty consecutive patients with active knee OA (diagnosed based on the American College of Rheumatology criteria for OA with radiographic confirmation) was performed. Concentrations of YKL-40 in serum and synovial fluid were measured by ELISA. US examinations - Gray scale (GS) US and Power Doppler (PD) US - of the knee was performed according to international guidelines. The suprapatellar, medial and lateral parapatellar recesses were scanned in each knee to evaluate synovial hypertrophy and vascularization. Results: Forty women (mean age $61.50 \pm 11.33$ years old) and 10 men (aged 68.50 \pm 6.60 years old) were enrolled. We found that the synovial level of the glycoprotein $(237.80 \pm 104.08 \mathrm{ng} / \mathrm{ml}) \mathrm{was}$ significantly higher compared to the serum concentration $(112.83 \pm 60.61 \mathrm{ng} / \mathrm{ml}, \mathrm{p}<0.001)$. The serum concentration in $\mathrm{OA}$ patients was higher comparing with age-matched healthy controls $(84.19 \pm 11.39 \mathrm{ng} / \mathrm{ml})(\mathrm{p}<0.05)$. A statistically significant association between YKL- 40 in synovial fluid and serum levels was shown. We determined a moderately positive linear relationship between the synovial level of the glycoprotein and the serum concentration. No association between the levels of inflammatory markers - erythrocyte sedimentation rate and C-reactive protein - and YKL-40 concentrations was detected. Our study revealed a strong relationship between YKL-40 in synovial fluid and GS US and feeble with PD US. YKL-40 correlated with inflammatory activity in knee joints and neovascularization detected by US. Conclusions: YKL-40 is involved in the pathogenesis of OA synovitis. Evaluation of YKL-40 levels in parallel with US might provide more sensitive and reliable information for the diagnosis and understanding of $\mathrm{OA}$.
\end{abstract}

Keywords: YKL-40; ultrasonography; knee osteoarthritis; biomarkers

\section{Introduction}

Osteoarthritis (OA) is a degenerative disease, leading to progressive loss of articular cartilage [1]. Despite progress in the diagnosis of the disease, there are no definitive biomarkers for early detection and monitoring of OA. Biological markers may provide a snapshot of cur-

Received 11.09.2017 Accepted 03.12.2017

Med Ultrason

2018, Vol. 20, No 1, 57-63

Corresponding author: Rositsa Karalilova, MD

Medical University of Plovdiv

University Hospital "Kaspela"

Clinic of Rheumatology

64 Sofia str., Plovdiv 4001, Bulgaria

Fax: +359 32648 807, Mobile: +359 878556560

E-mail: karalilova@hotmail.com rent events in joint tissues, allowing a rapid assessment of treatment [2].

The initial event in OA, from pathophysiologic perspective, is related to the changes in the cartilage (mild fibrillation of the cartilage surface). This event is followed by a response within the bone, typically in the subchondral bone, due to intracellular communications. Subsequently, sometimes, a subchondral bone cyst is formed. At this moment changes in the underlying metabolic activity within the bone and osteophyte formation can be found [3].

Inflammation has an important role in early OA and is the target for some treatment approaches, both pharmacologic or lifestyle changes related counseling [4]. The release of cartilage degradation products and the reactions within the subchondral bone may in part lead to 
proinflammatory mediators' synthesis, such as cytokines, neuropeptides, and obesity-related adipokines [5]. The inflammation of the synovial membrane that occurs in both early and late phases of OA is associated with alterations in the adjacent cartilage that are similar to those seen in rheumatoid arthritis [6].

Neovascularization, not just in the synovium but also in the bone itself, is another typical feature of disease pathogenesis. Angiogenesis has been demonstrated to be an important part of the early pathophysiologic process affecting both bone and cartilage in OA patients [7]. OA should be considered not as an inert disease, but as a metabolic reactive, reparative process that is initiated mechanically, and mediated biochemically, through inflammatory and neuropeptide pathways with additional angiogenic responses [8].

Ultrasonography (US) has been suggested to be a sensitive method that provides information regarding soft tissue, hyaline cartilage, bone surface, and articular effusion [9]. A small to moderate amount of synovitis and effusion were observed in OA (for example $-47 \%$ $-100 \%$ of the patients have synovitis and/or effusion in the symptomatic OA knee) [10]. US can detect minimal synovitis in a joint and to identify patients with a higher risk of progression [11].

Advantages of US assessments are the early detection of the structural changes of bone surface and the inflammatory changes inside the joint, and the excellent monitoring of subtle progression [10]. Synovitis or joint effusion detected by US correlated to pain in knee OA, magnetic resonance imaging, and arthroscopic findings [12]. The increased in synovial vascularization could be evaluated by both color and power Doppler (PD) US techniques. It was demonstrated that PD US findings correlate with histologic examination of proliferated synovia in patients with OA and rheumatoid arthritis (RA) [13]. US is used for early diagnosis of OA, to determine the type and extent of bone and cartilage damage, and for the detection of synovitis. This technique reveals the role of soft tissue as a source of pain and a contributing factor for disease progression and may facilitate treatment monitoring [11].

YKL-40 is a glycoprotein - a potential marker for active inflammatory process - with proven predictive value in a number of diseases that evolve with inflammation, remodeling of the extracellular matrix, or development of fibrosis [14]. The presence of YKL-40 in cartilage and synovium in OA patients could reflect the local disease activity. The functional role of the glycoprotein is not yet clear and no specific receptor is determined. It could be hypothesized that YKL-40 production, as part of the inflammatory response in articular chondrocytes, might modulate the cellular response to proinflammatory cytokines, acting to limit connective tissue degradation [15]. Several studies have investigated the presence of the glycoprotein in serum and synovial fluid of different joint diseases and 10-15-fold higher YKL-40 levels in synovial fluid were found [16-17]. Most published studies used radiographic examination to assess the disease process in joints. The data available are quite controversial [18-19].

The aim of our study was to analyze the level of the glycoprotein YKL-40 in patients with active knee OA and to search the possible correlations with local inflammation and US findings.

\section{Materials and methods}

\section{Patients}

Between March 2015 and May 2017, 50 consecutive patients with symptomatic active knee OA from the Rheumatology Clinic of University Hospital "Kaspela" were included in a prospective study. The study was approved by the local Ethics Committee and all patients provided written informed consent. The following data were recorded for each patient: age, gender, symptoms duration, radiographic stage according to the KellgrenLawrence scale, and therapy. The exclusion criteria were history of knee arthroplasty and local corticosteroid treatment in the last 8 weeks.

The diagnosis of knee OA was based on the American College of Rheumatology criteria for OA with radiographic confirmation [20]. All patients were clinically examined and oin the same day US examination, knee arthrocentesis, and blood tests (erythrocyte sedimentation rate [ESR] and C-reactive proteins [CRP] were performed. Patients were treated by analgetics, NSAIDs, chondroprotective agents.

\section{Control group}

We used the ranges of YKL-40 in the serum of healthy individuals found in previous investigations [21]. The control group consisted of 40 healthy subjects ( 24 women and $14 \mathrm{men}$ ). Clinical and routine hematological, biochemical and coagulation tests were completed to assess their health status.

\section{Measurement of YKL-40}

Concentrations of YKL-40, in serum and synovial fluid, were measured by ELISA, using commercial kits from Quidel, SA (YKL-40). The detection limit was 10 $\mu \mathrm{g} / \mathrm{ml}$. All samples were examined in duplicate.

\section{The US assessment}

The US investigation was performed using MyLab 7, Esaote, Italy machine with multifrequency linear probe (3-18 MHz). The grey scale (GS) US and PD US settings 
were adjusted to optimize image resolution and sensitivity to detect flow. Two ultrasonographers carried out a GS and PD US examination of both knees of each patient in a blind, independent, and consecutive manner. The suprapatellar, medial, and lateral parapatellar recesses were scanned and the following scanning planes were used for detection and scoring lesions: longitudinal plane for the suprapatellar recess, the femorotibial space (medially and laterally), transverse plane to the patella for the parapatellar recesses. The weight bearing surfaces of the femoral condyle were scanned in the suprapatellar region with the knee fully flexed. Scanning for fluid was performed both by dynamic examination and in the standard static position - supine in a flexion of $30^{\circ}$. GS US scoring was evaluated using a four-grade scale from 0 to 3 with the following subjective definitions for each category: grade 0 - absence of synovial thickening; grade 1 - mild synovial thickening; grade 2 - moderate synovial thickening; grade 3 - marked synovial thickening [22].

PD US was also evaluated using a four-grade scale from 0 to 3 with the following definitions for each category: grade 0 - no flow in the synovium; grade 1 - mild, single vessel signals; grade 2 - confluent vessel signals in the less than half of the area of the synovium; 3 - vessel signals in more than half of the area of the synovium
[22]. The highest found score for GS and PD US, regardless of the area where it is located, was taken into consideration [23].

\section{Statistical analysis}

The data were analyzed using the IBM SPSS 24 software package (SPSS, Chicago, Illinois, USA). Descriptive statistics are expressed in means, SD, minimum and maximum obtained scores. The main variables of interest (YKL-40 serum, YKL-40 synovial fluid, ESR, and CRP) were examined for normality through KolmogorovSmirnov $\mathrm{Z}$ test. The results showed that three of them (YKL-40 serum, YKL-40 synovial fluid, and CRP) were not normally distributed, $\mathrm{p}<0.05$. This determined the use of non-parametric tests, including Spearman Rho correlation and Wilcoxon Signed Ranks Test, to examine the relationships between them. In addition, possible correlations between the US data, GS US and PD US, and YKL-40 synovial fluid were analyzed through Spearman Rho correlation due to their ordinal or dichotomous nature. All statistical results are interpreted at the level of significance, alpha $(\alpha)=0.05$

\section{Results}

The demographic characteristics of the investigated patients are detailed in Table I.

Table I. Descriptive statistics of patients' data.

\begin{tabular}{|c|c|c|c|c|c|}
\hline Stratification & Variable & Mean & SD & Minimum & Maximum \\
\hline All patients & YKL-40 serum, ng/ml & 112.83 & 61 & 46 & 433 \\
\hline \multirow[t]{4}{*}{$(\mathrm{N}=50)$} & YKL-40 synovial fluid, $\mathrm{ng} / \mathrm{ml}$ & 237.8 & 104 & 102 & 577 \\
\hline & $\mathrm{ESR}, \mathrm{mm} / \mathrm{h}$ & 20.66 & 10.89 & 4 & 54 \\
\hline & CRP, mg/l & 6.24 & 3.04 & 2 & 14 \\
\hline & Age (years) & 62.50 & 10.88 & 40 & 93 \\
\hline Females & YKL-40 serum, ng/ml & 115.5 & 64.1 & 46.4 & 433.5 \\
\hline \multirow{4}{*}{$(\mathrm{N}=40)$} & YKL-40 synovial fluid, $\mathrm{ng} / \mathrm{ml}$ & 225.35 & 88.9 & 102.3 & 475.7 \\
\hline & $\mathrm{ESR}, \mathrm{mm} / \mathrm{h}$ & 22.3 & 11.2 & 5.0 & 54.0 \\
\hline & CRP, mg/l & 5.87 & 2.70 & 2.50 & 14 \\
\hline & Age (years) & 61.50 & 11.33 & 40 & 93 \\
\hline Males & YKL-40 serum, ng/ml & 102.2 & 45.1 & 46.4 & 176.6 \\
\hline \multirow[t]{4}{*}{$(\mathrm{N}=10)$} & YKL-40 synovial fluid, $\mathrm{ng} / \mathrm{ml}$ & 287.6 & 145.9 & 121.5 & 576.8 \\
\hline & $\mathrm{ESR}, \mathrm{mm} / \mathrm{h}$ & 15.20 & 7.55 & 4.0 & 27.0 \\
\hline & CRP, mg/l & 7.73 & 3.79 & 2.0 & 12.80 \\
\hline & Age (years) & 68.50 & 6.62 & 54 & 78 \\
\hline$\leq 50$ years & YKL-40 serum, ng/ml & 108.85 & 14.21 & 99.6 & 137.36 \\
\hline \multirow[t]{4}{*}{$(\mathrm{N}=6)$} & YKL-40 synovial fluid, $\mathrm{ng} / \mathrm{ml}$ & 202.97 & 35.45 & 131.4 & 224.43 \\
\hline & $\mathrm{CYE}, \mathrm{mm} / \mathrm{h}$ & 27.16 & 13.18 & 14.0 & 46.0 \\
\hline & $\mathrm{CRP}, \mathrm{mg} / \mathrm{l}$ & 4.50 & 1.22 & 3.0 & 6.0 \\
\hline & Age & 43.88 & 2.40 & 40 & 47 \\
\hline$>50$ years & YKL-40 serum, ng/ml & 113.37 & 64.50 & 46.4 & 433.46 \\
\hline \multirow[t]{4}{*}{$(\mathrm{N}=44)$} & YKL-40 synovial fluid, $\mathrm{ng} / \mathrm{ml}$ & 244.55 & 109.57 & 102.3 & 576.76 \\
\hline & $\mathrm{CYE}, \mathrm{mm} / \mathrm{h}$ & 19.77 & 10.403 & 4.0 & 54.0 \\
\hline & CRP, mg/l & 6.48 & 3.10 & 2.0 & 14.0 \\
\hline & Age & 65.50 & 8.75 & 53 & 93 \\
\hline
\end{tabular}

$\mathrm{SD}$ - standard deviation; $\mathrm{N}$ - number of patients 
Serum and synovial fluid of YKL-40 levels and laboratory parameters - (ESR and CRP)

We found no significant association between age and the four variables: YKL-40 serum, YKL-40 synovial fluid, ESR, and CRP (all p>0.05). A similar trend was observed for gender, which showed no significant correlation with the variables of interest: YKL-40 serum, YKL-40 synovial fluid, ESR, and CRP, (all p>0.05).

No relationship between laboratory parameters (ESR and CRP) and YKL-40 levels in serum and synovial fluid was detected in the patients' group.

The mean serum concentration of YKL-40 in knee OA patients was $112.83 \pm 61 \mathrm{ng} / \mathrm{ml}$, which is higher compared to that of age-matched healthy controls $(84.19 \pm 11.39 \mathrm{ng} /$ ml) $(\mathrm{p}<0.05)$.

We found that the synovial level of YKL-40 $(237.80 \pm 104.08 \mathrm{ng} / \mathrm{ml})$ was significantly higher compared to the serum concentration $(112.83 \pm 60.61 \mathrm{ng} / \mathrm{ml})$, $\mathrm{p}<0.001$. A statistically significant correlation between YKL- 40 in synovial fluid and serum levels was determined $r_{s}=0.532, p<0.001$. We also found a moderately positive linear relationship between the synovial level of the glycoprotein and the serum concentration $\left(\mathrm{r}_{\mathrm{s}}=0.238\right)$.

\section{Synovial YKL-40 levels and US findings}

The US results are summarized in Table II.

Analyzing the relationship between YKL-40 level in synovial fluid and US findings, a statistically significant and rather strong correlation between GS US and YKL-

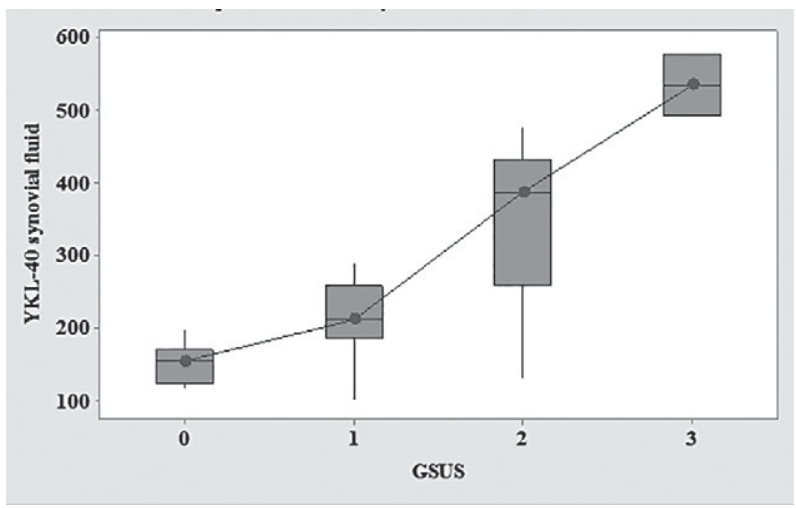

Fig 1. Box plots of YKL-40 synovial fluid depending of grey scale ultrasonography (GSUS) levels.
40 in synovial fluid, $\left(r_{s}=0.690, p<0.001\right)$ was found. The relationship between YKL-40 synovial fluid and PD US was statistically significant, but feeble, $r_{s}=0.303$, $\mathrm{p}=0.033$.

PD US examination determined 45 patients with no signal and 5 patients with score 1 . In a more varied sample group the association may be stronger. A sharp increase in the mean values of the synovial level of the glycoprotein was observed in patients with higher score in GS US (fig 1).

\section{Discussion}

$\mathrm{OA}$ is the most common disease of the joint, resulting in physical disability. It is characterized by the loss of the articular cartilage and alterations in the subchondral bone [24]. The reasons behind OA progression remain undefined and understanding molecular pathogenesis of the disease is still in progress. A growing number of investigations have found that the recently discovered glycoprotein YKL-40 could be involved in the inflammatory joint process. YKL-40, the human chitinase 3-like-1 protein, is linked to different mediators of inflammation and cartilage damage [25]. It has been shown that YKL-40 synthesis is related to joint inflammation, tissue remodeling, and alteration of cartilage in OA joint [26].

YKL-40 has no enzymatic activity and no specific receptor for it is known. It has been proposed that proteoglycans and collagens are potential ligands of the glycoprotein. These components are important part of the cartilage extracellular matrix and their production and alteration is influenced by YKL-40 [27]. Researchers suppose that YKL-40 interacts with part of the syndecan receptor, belonging to a family of cell surface receptors, involved in synovial inflammation and cartilage degradation [28].

Despite the intensive interest and investigations in recent years, the role of YKL-40 in OA remains unclear.

In the current study, higher levels of YKL-40 in the synovial fluid of OA patients compared to serum concentrations were detected. The elevation of synovial fluid levels of YKL-40 in comparison with serum levels was determined also in previous published studies $[16,17,29]$.

Table II. Serum and synovial fluid YKL-40 and US findings

\begin{tabular}{llllll}
\hline Number of patients & $\begin{array}{l}\text { YKL-40 in serum } \\
(\mathbf{m e a n} \pm \mathbf{S D})\end{array}$ & $\begin{array}{l}\text { YKL-40 in synovial fluid } \\
(\mathbf{m e a n} \pm \mathbf{S D})\end{array}$ & $\begin{array}{l}\text { GS US } \\
\text { score }\end{array}$ & \multicolumn{2}{c}{ PD US score } \\
\hline 9 & $81.76 \pm 33.64$ & $151.40 \pm 26.70$ & 0 & 9 & 0 \\
31 & $107.56 \pm 30.08$ & $214.54 \pm 45.52$ & 1 & 31 & 0 \\
8 & $166.94 \pm 123.50$ & $350.86 \pm 117.48$ & 2 & 5 & 3 \\
2 & $117.62 \pm 63.59$ & $535.00 \pm 59.06$ & 3 & 0 & 2 \\
\hline
\end{tabular}

SD - standard deviation 
Serum concentrations in OA patients were reported to be increased compared to those in healthy individuals $[30,31]$, as we found also in our study.

There are other studies focused on YKL-40 in RA showing that their concentrations in both serum and synovial fluid reflected active inflammation and intensive angiogenesis in synovial membrane $[21,32]$. In this study we observed that the higher grade in GS US correlated with significantly increased YKL-40 levels in synovial fluid of OA patients. US examination detected cartilage lesions, joint capsule hypertrophy and fibrosis, synovial hypertrophy and joint effusion. Traditionally, conventional radiography is used to diagnose the changes of knee OA. However, early changes are difficult to assess and this technique is not capable of visualizing the local inflammatory component. US is an imaging technique that is able to illustrate both bone structural changes and inflammatory alterations within the joint [20].

Musculoskeletal US is an established technique for assessing the progression of knee joint $\mathrm{OA}$ and can be used as an imaging biomarker [33].

Of all US changes that can be found in an OA, we studied the following aspects of knee inflammation GS US synovial hypertrophy and effusion and the presence of synovial PD US,. As in other studies we have evidenced that YKL-40 correlates with inflammatory changes in the joint [21]. In addition, we investigated YKL-40 in the synovial fluid, which suggest secondary inflammatory changes in the osteoarthritis joint.

A high prevalence of both synovial hypertrophy and effusion was found. Regarding the synovial PD US signal, its low prevalence in our patients questions the reliability of this parameter in OA. According to some researchers synovial neovascularisation is not a prominent feature in OA [30].

Our study revealed that the relationship between levels of YKL-40 in synovial fluid and PD US was statistically significant, but feeble. However, this result was expected due to the limited number of PD US positive patients. It is shown that YKL-40 stimulates neovascularization and acts synergistically with VEGF [29]. So, we might suppose that YKL-40 also illustrates the vascularization of the synovial membrane in knee OA patients similar to PD US. Within OA joints, YKL-40 is secreted into the synovial fluid by chondrocytes, activated macrophages and synovial fibroblasts [16,31]. Different investigations have demonstrated that osteoblasts and osteocytes present in osteophytes expressed YKL-40 $[14,18]$. Thus, our finding of higher YKL-40 levels in synovial fluid reflects OA changes in the joint tissue. It is supposed that chondrocytes are the major player in the cartilage degradation [25].
We found no associations between YKL-40 levels in serum and synovial fluid and CRP and ESR. In contrast, we reported a significant relationship between these parameters but in patients with RA [21]. CRP and ESR are conventional markers that provide information about disease activity, but are not predictive alone to be used for treatment decision making [32].

Our results suggest that YKL-40 is a more suitable biomarker functionally associated with the development of OA, compared to routinely used inflammation markers. Other researchers reported that chondrocytes of human OA cartilage secreted inflammation associated YKL-40 [34].

Furthermore, the glycoprotein levels in synovial fluid, but not in serum were positively related to pain and physical activity limitation in knee OA [35]. Szychlinska et al proposed that YKL-40 might be considered as a potential natural agent providing therapeutic effects in joint inflammation [36].

The molecular changes in OA are intensively investigated but its basic mechanisms are still unknown. We could conclude that YKL-40 is a more sensitive and reliable marker reflecting a certain grade of morphological cartilage degeneration compared to ESR and CRP. Our findings support the role of YKL-40 in the pathogenesis of OA synovitis. It is a challenge to explore the role of this biomarker as a potential independent tool for the diagnosis and understanding of OA.

Our study had some limitations. We could not examine the effect of medical treatment on YKL-40 levels since our patients were on heterogeneous medication. Prospective and comparative studies are required in the future. Other limitations of the study are the limited number of patients, the lack of inter- and intra-observer agreement for US examination and the lack of comparison of US findings with other imaging techniques.

\section{Conclusions}

In conclusion, YKL-40 is involved in the pathogenesis of OA synovitis. Evaluation of YKL-40 levels in parallel with US might provide more sensitive and reliable information for the diagnosis and understanding of $\mathrm{OA}$

Acknowledgements: The study is supported by grant NO 02/2015 from the Medical University - Plovdiv and grant DUNK 01/2009 from the Bulgarian Ministry of Education and Science.

Conflict of interest: none 


\section{References}

1. McCarthy R. New concepts in moderate to severe osteoarthritis treatment. A clinical practice assessment. Osteoarthritis Cartilage 2017;25:S178.

2. Bijlsma JW, Berenbaum F, Lafeber FP. Osteoarthritis: an update with relevance for clinical practice. Lancet 2011;377:2115-2126.

3. Li G, Yin J, Gao J, Cheng TS, Pavlos NJ, Zhang C, Zheng MH. Subchondral bone in osteoarthritis: insight into risk factors and microstructural changes. Arthritis Res Ther 2013;15:223.

4. Sellam J, Berenbaum F. The role of synovitis in pathophysiology and clinical symptoms of osteoarthritis. Nat Rev Rheumatol 2010;6:625-635.

5. Kapoor M, Martel-Pelletier J, Lajeunesse D, Pelletier J, Fahmi H. Role of proinflammatory cytokines in the pathophysiology of osteoarthritis. Nat Rev Rheumatol 2011;7:33-42.

6. Johnson VL, Hunter DJ. The epidemiology of osteoarthritis. Best Pract Res Clin Rheumatol. 2014;28:5-15.

7. Lluch E, Torres R, Nijs J, Van Oosterwijck J. Evidence for central sensitization in patients with osteoarthritis pain: a systematic literature review. Eur J Pain 2014;18:1367-1375.

8. Bruyère $\mathrm{O}$, Cooper $\mathrm{C}$, Pelletier $\mathrm{JP}$, et al. An algorithm recommendation for the management of knee osteoarthritis in Europe and internationally: a report from a task force of the European Society for Clinical and Economic Aspects of Osteoporosis and Osteoarthritis (ESCEO). Semin Arthritis Rheum 2014;44:253-263.

9. Batalov A, Kuzmanova SI, Penev DP. Ultrasonographic evaluation of knee joint cartilage in rheumatoid arthritis patients. Folia Medica 2000;4:23-26.

10. Kristoffersen H, Torp-Pedersen S, Terslev L. Indications of inflammation visualized by ultrasound in osteoarthritis of the knee. Acta Radiol 2006;47:281-286.

11. Moller D, Bong E, Naredo E, et al. Ultrasound in the study and monitoring of osteoarthritis. Osteoarthritis Cartilage 2008;16 Suppl 3:S4-S7.

12. De Miguel Mendieta E, Cobo Ibanez T, Uson Jaeger J, Bonilla Hernan G, Martin Mola E. Clinical and ultrasonographic findings related to knee pain in osteoarthritis. Osteoarthritis Cartilage 2006;14:540-544.

13. Walther M, Harms H, Krenn V, Radke S, Faehndrich T, Gohlke F. Correlation of the power Doppler sonography with the synovial tissue of the knee joint in patients with osteoarthritis and rheumatoid arthritis. Arthritis Rheum 2001;44:331-338.

14. Johansen JS. Studies on serum YKL-40 as a biomarker in diseases with inflammation, tissue remodelling, fibroses and cancer. Dan Med Bull 2006;53:172-209.

15. Johansen JS, Olee T, Price PA, Hashimoto S, Ochs RL, Lotz M. Regulation of YKL-40 production by human articular chondrocytes. Arthritis Rheum 2001;44:826-837.

16. Volck B, Johansen JS, Stoltenberg M, et al. Studies on YKL-40 in knee joints of patients with rheumatoid arthritis and osteoarthritis. Involvement of YKL-40 in the joint pathology. Osteoarthritis Cartilage 2001;9:203-214.
17. Conrozier T, Carlier MC, Mathieu P, et al. Serum levels of YKL-40 and C reactive protein in patients with hip osteoarthritis and healthy subjects: a cross sectional study. Ann Rheum Dis 2000;59:828-831.

18. Zivanovic S, Rackov LP, Vojvodic D, Vucetic D. Human cartilage glycoprotein 39-biomarker of joint damage in knee osteoarthritis. Int Orthop 2009;33:1165-1170.

19. Garnero P1, Piperno M, Gineyts E, Christgau S, Delmas PD, Vignon E. Cross sectional evaluation of biochemical markers of bone, cartilage, and synovial tissue metabolism in patients with knee osteoarthritis: relations with disease activity and joint damage. Ann Rheum Dis 2001;60:619-626.

20. Altman R, Asch E, Bloch D, et al. Development of criteria for the classification and reporting of osteoarthritis. Classification of the osteoarthritis of the knee. Diagnostic and Therapeutic Criteria Committee of the American Rheumatism Association. Arthritis Rheum 1986;29:1039-1049.

21. Kazakova M, Batalov A, Deneva T, Mateva N, Kolarov Z, Sarafian V. Relationship between sonographic parameters and YKL-40 levels in rheumatoid arthritis. Rheumatol Int 2013;33:341-346.

22. Szkudlarek M, Court-Payen M, Jacobsen S, Klalund M, Thomsen HS, Ostergaard M. Interobserver agreement in ultrasonography of the finger and toe joints in rheumatoid arthritis. Arthritis Rheum 2003;48:955-962.

23. Mandl P, Naredo E, Wakefield RJ, Conaghan PG, D'Agostino MA; OMERACT Ultrasound Task Force. A systematic literature review analysis of ultrasound joint count and scoring system to assess synovitis in rheumatoid arthritis according to the OMERACT filter. J Rheumatol 2011;38:2055-2062.

24. Giunta S, Castorina A, Marzagalli R, et al. Ameliorative effects of PACAP against cartilage degeneration. Morphological, immunohistochemical and biochemical evidence from in vivo and in vitro models of rat osteoarthritis. Int $\mathrm{J}$ Mol Sci 2015;16:5922-5944.

25. Väänänen T, Koskinen A, Paukkeri E, et al. YKL-40 as a novel factor associated with inflammation and catabolic mechanisms in osteoarthritic joints. Mediators Inflamm 2014;2014:215140.

26. Di Rosa M, Malaguarnera G, de Gregorio C, Drago F, Malaguarnera, L. Evaluation of CHI3L-1 and CHIT-1 expression in differentiated and polarized macrophages. Inflammation 2013;36:482-492.

27. Shao R. YKL-40 acts as an angiogenic factor to promote tumor angiogenesis. Front Physiol 2013;4:122.

28. Pap T, Bertrand J. Syndecans in cartilage breakdown and synovial inflammation. Nat Rev Rheumat 2013;9:43-55.

29. Johansen JS, Jensen HS, Price PA. A new biochemical marker for joint injury. Analysis of YKL 40 in serum and synovial fluid. Br J Rheumatol 1993;32:949-955.

30. Bevers K, Zweers MC, van den Ende CH, et al. Ultrasonographic analysis in knee osteoarthritis: evaluation of interobserver reliability. Clin Exp Rheumatol 2012;30:673-678.

31. Kawasaki M, Hasegawa Y, Kondo S, Iwata H. Concentration and localization of YKL-40 in hip joint diseases. J Rheumatol 2001;28:341-345. 
32. Atzeni F, Talotta R, Masala I, Bongiovanni S, Boccassini L, Sarzi-Puttini P. Biomarkers in Rheumatoid Arthritis. Isr Med Assoc J 2017;19:512-516.

33. Oo WM, Bo MT. Role of Ultrasonography in Knee Osteoarthritis. J Clin Rheumatol 2016;22:324-329.

34. Einarsson JM, Bahrke S, Sigurdsson BT, et al. Partially acetylated chitooligosaccharides bind to YKL-40 and stimulate growth of human osteoarthritic chondrocytes. Biochem Biophys Res Commun 2013;434:298-304.
35. Guan J, Liu Z, Li F, et al. Increased synovial fluid YKL-40 levels are linked with symptomatic severity in knee osteoarthritis patients. Clin Lab 2015;61:991-997.

36. Szychlinska MA, Trovato FM, Di Rosa M, et al. Co-expression and co-localization of cartilage glycoproteins CHI3L1 and lubricin in osteoarthritic cartilage: morphological, immunohistochemical and gene expression profiles. Int J Mol Sci 2016:17:359. 